\title{
Deutsches Jahrbuch für Volkskunde
}

\author{
Herausgegeben vom Institut für deutsche Volkskunde \\ an der Deutschen Akademie der Wissenschaften zu Berlin \\ durch Hermann Strobach \\ Begründet von Wilhelm Fraenger und Wolfgang Steinitz
}

\begin{abstract}
REDAKTION
Dr. Wolfgang Jacobeit (Besprechungen) - Dr. Doris Stockmann (Mitteilungen und Berichte) - Dr. Hermann Strobach (Leitung, Abhandlungen) - Herta Uhlrich
\end{abstract}

(Bücherschau)

\begin{abstract}
REDAKTIONSBEIRAT
Dr. Karl Baumgarten, Rostock - Dr. Gisela Burde-Schneidewind, Berlin - Prof. Dr. Paul Nedo, Berlin - Dr. Reinhard Peesch, Berlin - Dr. Friedrich Sieber, Dresden Dr. Erich Stockmann, Berlin - Dr. Günther Voigt, Potsdam - Dr. Rudolf Weinhold, Dresden
\end{abstract}

INHALTSVERZEICHNIS

\section{ABHANDLUNGEN}

Oskár Elschek, Bratislava, und Erich Stockmann, Berlin: Zur Typologie der Volksmusikinstrumente . . . . . . . . . . . . . . . . . 225

ERIK Hühns, Berlin: Der Berliner Totentanz . . . . . . . . . . . . . . 235

DESMOND Mc CourT, Londonderry: Hausformen in einem kulturellen Kontaktgebiet Nordirlands . . . . . . . . . . . . . . . . . . . 245

HANS-JÜRGEN RACH, Berlin: Mitteldeutsche Ernhäuser mit einer Abseite in Brandenburg . . . . . . . . . . . . . . . . . 261

WERNER RADIG, Berlin: Frühgeschichtliche Grabungsbefunde und rezente ländliche Altbauten. . . . . . . . . . . . . . . . . . . . . 271 Waltraud Woeller, Potsdam: Die Sage vom Fliegenden Holländer . . . . 292 Fortsetzung des Inhaltsverzeichnisses auf der 3 . Umschlagseite 


\title{
DEUTSCHES JAHRBUCH FÜR VOLKSKUNDE
}

Herausgegeben vom Institut für deutsche Volkskunde an der Deutschen Akademie der Wissenschaften zu Berlin durch Hermann Strobach

Begründet von Wilhelm Fraenger und Wolfgang Steinitz

\author{
Vierzehnter Band \\ Jahrgang 1968
}

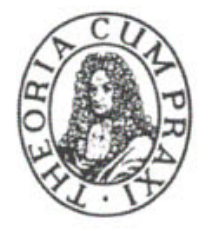

A K A D E I E-VER LA G B ERLIN 


\section{REDAKTION}

Dr. Wolfgang Jacobeit (Besprechungen) - Dr. Doris Stockmann (Mitteilungen und Berichte) - Dr. Hermann Strobach (Leitung, Abhandlungen) - Herta Uhlrich

(Bücherschau)

\section{REDAKTIONSBEIRAT}

Dr. Karl Baumgarten, Rostock - Dr. Gisela Burde-Schneidewind, Berlin - Prof. Dr. Paul Nedo, Berlin - Dr. Reinhard Peesch, Berlin - Dr. Friedrich Sieber, Dresden Dr. Erich Stockmann, Berlin - Dr. Günther Voigt, Potsdam - Dr. Rudolf Weinhold, Dresden

Herausgeber: Institut für deutsche Volkskunde an der Deutschen Akademie der Wissenschaften zu Berlin, Schriftleitung: Dr. Hermann Strobach, 108 Berlin 8, Unter den Linden 8; Fernsprecher: 2004 81. Verlag: Akademie-Verlag GmbH, Berlin W 8, Leipziger Str. 3-4; Fernsprecher: Sammelnummer 22 044x. Telex-Nr. or 1773 . Postscheckkonto: Berlin 35021. Das Deutsche Jahrbuch für Volkskunde erscheint jährlich. Bestellnummer dieses Bandes: 1034/XIV. VEB Druckhaus „Maxim Gorki“, 74 Altenburg. Veröffentlicht unter der Lizenzaummer 1313 des Presseamtes beim Vorsitzenden des Ministerrates der Deutschen 


\section{INHALTSVERZEICHNIS}

\section{ABHANDLUNGEN}

Mrhar Pop, Bukarest: Der formelhafte Charakter der Volksdichtung . . . . I

Reinhard Peesch, Berlin: Benennungen und Bennenungsmotive zu einem Gerätetyp . . . . . . . . . . . . . . . . 16

UlRICH BENTZIEN, Rostock: Tiereigennamen. Untersucht an einem Quellenfund aus Mecklenburg . . . . . . . . . . . . . 39

HEINZ RosenkranZ, Jena: Ortsnecknamen und Einwohnernamen im Thüringischen ....................... s6

Hermann Bock und Heinz Rosenkranz, Jena: Zur Technik und Terminologie der Langholzflößerei auf der thüringischen Saale . . . . . . . . 84

OSKÁR ElsCheK, Bratislava, und ERICH StockMANN, Berlin: Zur Typologie der Volksmusikinstrumente . . . . . . . . . . 22s

ERIK HüHNs, Berlin: Der Berliner Totentanz. . . . . . . . . . 235

DESMOND MCCouRT, Londonderry: Hausformen in einem kulturellen Kontaktgebiet Nordirlands . . . . . . . . . . . . . . . 245

HANS-JÜRGEN RACH, Berlin: Mitteldeutsche Ernhäuser mit einer Abseite in Brandenburg ..................... 26r

WeRner RAdIG, Berlin: Frühgeschichtliche Grabungsbefunde und rezente ländliche Altbauten. Zum Forschungsstand . . . . . . . . 27r

Waltraud WoEller, Potsdam: Die Sage vom Fliegenden Holländer . . . . 292

\section{MITTEILUNGEN UND BERICHTE}

Rudolf WeInhold, Dresden: Alfred Fiedler 6s Jahre . . . . . . . . . . . . . 99 Ulrich Bentzien, Rostock: Arno Schmidtf . . . . . . . . . . . . . . . . . 100 Winfried SchrammeK, Leipzig: Zweite Internationale Arbeitstagung für die Erforschung der Volksmusikinstrumente Europas . . . . . . . . . . . . . ror

Vilmos Vorgt, Budapest: Fünfter Kongreß der Association Internationale de Littérature Comparée vom 30. August bis s. September 1967 in Beograd . . . . . . . I06

IzALIJ ZemcovskiJ, Leningrad: Die Musikfolkloristik in der Russischen SFSR. Ein Literaturbericht von $1946-1967 \ldots$. . . . . . . . . . . . . . . 108 
KarL Spangenberg, Jena: Rotwelsch aus der Mitte des 19. Jahrhunderts. Ein Fundbericht. . . . . . . . . . . . . . . . . . . . IIs

Siegfried Kube, Dresden: Friedrich Sieber 75 Jahre . . . . . . . . . . 315

Wolfgang Jacobeit, Berlin, und Paul Nowotny, Bautzen: Paul Nedo 6o Jahre . . 316

Jochen Helbig, Dresden: Werner Radig 65 Jahre . . . . . . . . . . . . . . . 318

John Granlund, Stockholm: Sigurd Erixon zum Gedenken . . . . . . . . . . . 320

JAN StĘSZEwSKI, Warschau: Nachruf auf Marian Sobieski . . . . . . . . . . . 322

Gisela BURdE-SChNEIDEwind, Berlin: Kolloquium über volkskundliche Probleme des 19./20. Jahrhunderts - 4. bis 6. Dezember 1967 in Bad Saarow bei Berlin . . . . 323

Doris StockmanN, Berlin: Erste Arbeitstagung der Studiengruppe zur Erforschung und Edition älterer Volksliedquellen des International Folk Music Council (IFMC) 326

Doris Stockmann, Berlin: 3. Arbeitskonferenz der Study Group of Folk Music Systematization des IFMC . . . . . . . . . . . . . . . . . . . . . . . . 328

PAul Nowotny, Bautzen: Zur Methodik der soziologisch-ethnographischen Erforschung der Gemeinde Radibor - Kreis Bautzen . . . . . . . . . . . . . . . 329

Ants Virres, Tallinn: Uber die ethnographische Arbeit in der Estnischen SSR . . . 333

Juhani U. E. Lehtonen, Helsinki: Dorfforschung in Finnland . . . . . . . . . . 338

Alfred Fiedler, Dresden: Vom Werkzeug der Zimmerleute im Elbsandsteingebirge um die Wende vom 19. zum 20. Jahrhundert - Ein Beitrag zur Erforschung des handwerklichen Arbeitsgerätes . . . . . . . . . . . . . . . . 34I

ReINHARD PEesch, Berlin: Volkskunstmotive auf der Schreibmaschine geschrieben Erna Dietzel zum Gedächtnis . . . . . . . . . . . . . . . . 353

\section{BUCHERSCHAU}

Herta Uhlrich, Berlin: Museen und Museumskunde in deutschsprachigen Veröffentlichungen $1956-1967$. . . . . . . . . . . . . . . . . . . . . . . 124

N. S. PolıščuK, Moskau: Literatur zur volkskundlichen Gegenwartsforschung. UdSSR. Russische ethnographische Veröffentlichungen 1958-1967 . . . . . 355

Herta Uhlrich, Berlin: Fischerei und Jagd in deutschsprachigen Veröffentlichungen 1959-1968 . . . . . . . . . . . . . . . . . . . . . 363

\section{BESPRECHUNGEN}

Populus revisus. Beiträge zur Erforschung der Gegenwart (Hermann Strobach) . . 146 SIGFRID Svensson, Introduktion till folklivsforskningen (Einführung in die Volkskundeforschung) (Gösta Berg) . . . . . . . . . . . . . . . . . . I49

EMERICH Francis, Ethnos und Demos. Soziologische Beiträge zur Volkstheorie (Paul Nowotny). . . . . . . . . . . . . . . . . . . . . . . . Iso

Paul Nedo, Grundriß der sorbischen Volksdichtung (Hermann Strobach) . . . . . Is 2 Słownik folkloru polskiego (Wörterbuch der polnischen Folklore) Red. von JuliaN KRZYŻANOWSKI (Bernd Schöne) . . . . . . . . . . . . . . . . Is6

75 Jahre Museum für Volkskunde zu Berlin 1889-1964. Festschrift (Ulrich Bentzien) I 8 Werte der deutschen Heimat (Rudolf Weinhold). . . . . . . . . . . . . . . 160 Etnologia y Folklore, hg. v. Instituto de Etnologia y Folklore der kubanischen Akad. der Wiss. zu La Habana (Ursula Schlenther) . . . . . . . . . . . . . . . 16I 
LEOPOLD Schmidt, Volkskunde von Niederösterreich, Bd. I (Rudolf Weinhold) . . 162

Horňácko (Leben und Kultur des Volkes im mährisch-slowakischen Grenzgebiet im Raum der Weißen Karpaten (Blasius Nawka) . . . . . . . . . . . . . . . . 164

Helmut Bleiber, Zwischen Reform und Revolution (Hainer Plaul) . . . . . . . . 166

The Cambridge Economic History of Europe (Ulrich Bentzien) . . . . . . . . . . ${ }^{6} 67$

BRoniseava Kopczy ŃSKa-JaWorsKa, Stosunki gospodarczo-spoleczne w tradycyjnej kulturze Karpat Północnych (Wirtschaftlich-gesellschaftliche Verhältnisse in der traditionellen Kultur der Nordkarpaten) (Bernd Schöne) . . . . . . . . . . . . 170

Hunting and Fishing. Nordic Symposium on Life in a Traditional Hunting and Fishing Milieu in Prehistoric Times and up to the Present Day. Hg. Harald Hvarfaner (Holger Rasmussen)

Walter Bogsch, Der Marienberger Bergbau seit der zweiten Hälfte des I6. Jahrhunderts (Helmut Wilsdorf) . . . . . . . . . . . . . . . . . . . I73

JOSEPH KLERSCH, Volkstum und Volksleben in Köln (Friedrich Sieber) . . . . . . 175

LEOPOLD Schmidt, Volksglaube und Volksbrauch. Gestalten. Gebilde. Gebärden (Siegfried Kube) . . . . . . . . . . . . . . . . . . . . . . . . . . . ${ }^{176}$

Dieter Lutz, Volksbrauch und Sprache. Die Benennung von Phänomenen der Winterund Frühlingsbräuche Südwestdeutschlands (Helmut Schönfeld). . . . . . . . . 177

Wolfgang BRÜcKNeR, Bildnis und Brauch. Studien zur Bildfunktion der Effigies (Friedrich Sieber) . . . . . . . . . . . . . . . . . . . 179

Elke Stein, Hungrige speisen (Ute Mohrmann) . . . . . . . . . . . . . . . I80

Dieter Dünninger, Wegsperre und Lösung (Friedrich Sieber) . . . . . . . . . I8I

ERIKA Weltr, Taufbräuche im Kanton Zürich (Ders.) . . . . . . . . . . . . . . 183

Werner LenK, Das Nürnberger Fastnachtspiel des I5. Jahrhunderts (Hans-Ulrich und Monika Roller). . . . . . . . . . . . . . . . . . . . . . 183

Hermann Fischer, Volkslied, Schlager, Evergreen (Vladimir Karbusický) . . . . . 186

Des Knaben Wunderhorn. Alte deutsche Lieder. Hg. von Hans-Günther Thalheim (Hermann Strobach) . . . . . . . . . . . . . . . . . I89

HeinRICH W. Schwab, Sangbarkeit, Popularität und Kunstlied (Hermann Strobach) I9I

Georges-Denis Zimmermann, Irish Political Street Ballads and Rebel Songs 1780 to 1900 (Brigitte Emmrich) . . . . . . . . . . . . . . . . . 193

Danish Ballads and Folk Songs. Hg. Erik Dal (Dies.) . . . . . . . . . . . . . . 196

JAN RAUPP, Sorbische Musik (Erich Stockmann) . . . . . . . . . . . . . . . . 197

Werner Bachmann, Die Anfänge des Streichinstrumentenspiels (Ernst Emsheimer) 198

JAN LING, Nyckelharpan. Studier i ett folkligt musikinstrument (Erich Stockmann) . 201

Lutz RöHrich, Gebärde - Metapher - Parodie. Studien zur Sprache und Volksdichtung (Siegfried Neumann) . . . . . . . . . . . . . . . . . . 204

Odell ShePARd, The Lore of the Unicorn (Friedrich Sieber) . . . . . . . . . . 205

Gerhard Heilfurth unter Mitarb. v. Ina-Maria Greverus, Bergbau und Bergmann in der deutschsprachigen Sagenüberlieferung Mitteleuropas. Bd. x. - Quellen. (Gisela Burde-Schneidewind, Helmut Wilsdorf) .

Schwäbische Volkssagen vom Schwarzwald zum Allgäu - vom Taubergrund zum Bodensee. Hg. v. F. H. Schmidt-EbHausen (Christiane Agricola) . . . . . . . . 2 II Novellistica Italo-albanese. Testi orali raccolti dal Prof. Luca Perrone (Wilfried Fiedler) 212 Andreas Tietze, The Koman Riddles and Turkic Folklore (Friedmar Geißler) . . . 213 
Oskar Reichmann, Der Wortschatz der Siegerländer Landwirtschaft und Haubergswirtschaft (Heinz Gebhardt) . . . . . . . . . . . . . . . . 214

RolF MƯller, Die Synonymik von „Peitsche“. Semantische Vorgänge in einem Wortbereich (Karl Spangenberg) . . . . . . . . . . . . . . . . 210

Ole HöjRup, Landbokvinden. Rok og Kärne. Grovbröd og Vadmet (Gösta Berg) 218

Unsere Liebe, unsere Kunst der DDR. Katalog zur Ausstellung des bildnerischen Volksschaffens anläßlich der 9. Arbeiterfestspiele Dresden 1967 (Ute Mohrmann) . 219

Hermann Phleps, Alemannische Holzbaukunst. Hg. u. bearb. von ERNSt Mix (Karl Baumgarten)

FRANZ LIPP, Oberösterreichische Stuben. Bäuerliche und bürgerliche Innenräume Möbel und Hausgerät (Gislind M. Ritz) . . . . . . . . . . . . . . . . . . .

IGOR VAHRos, Zur Geschichte und Folklore der großrussischen Sauna (Karl Baumgarten)

Helmut Hanke, Kultur und Lebensweise im sozialistischen Dorf (Ute Mohrmann) . 374

KARL-Sigismund KRAMER, Volksleben im Hochstift Bamberg und im Fürstentum Coburg (1500-1800) (Rudolf Weinhold) . . . . . . . . . . . 375

GERD SPIES, Braunschweiger Volksleben nach Bildern von Carl Schröder (1802 bis 1867) (Siegfried Kube) . . . . . . . . . . . . . . . . . 378

Nils-Arvid Bringéus, Gunnar Olof Hyltén-Cavallius som etnolog. En studie kring Wärend och wirdarne (Hainer Plaul) . . . . . . . . . . . . . . . . 379

Hermann Aubin, Theodor Frings, Josef Müller, Kulturströmungen und Kulturprovinzen in den Rheinlanden. Geschichte, Sprache, Volkskunde (Reinhard Peesch) 380 JöRG EhNI, Das Bild der Heimat im Schullesebuch (Hainer Plaul) . . . . . . . . . 38I

OLOF HASSLÖF, En släkt och dess skepp (Ein Geschlecht und dessen Schiffe) (Wolfgang Rudolph) . . . . . . . . . . . . . . . . . . 383

Lothar Pretzell, Kostbares Volksgut. Aus dem Museum für Deutsche Volkskunde Berlin (West) (Erika Karasek) . . . . . . . . . . . . . . . . . . . . . . 384

Der Steirische Bauer - Leistung und Schicksal von der Steinzeit bis zur Gegenwart. Katalog der Steirischen Landesausstellung Hg. Frirz Posch (Dies.) . . . . . . . 385

Volkskundliche Sammlungen. Bauernkultur in Mecklenburg II: Das Mobiliar. Hg. v. d. Direktion d. Staatl. Museums Schwerin (Siegfried Neumann) . . . . . . 386

AMA - Acta Museorum Agriculturae (Vestnik sel'skochozjajstvennych muzeev) Jg. I. 2 (Ulrich Bentzien) . . . . . . . . . . . . . . . . . . . . . . . . . 387

Rostocker Beiträge. Regionalgeschichtliches Jahrbuch der mecklenburgischen Seestädte. Hg. v. d. Stadtarchiven Rostock und Wismar durch JoHanNes Lachs. Bd. I (Siegfried Neumann) . . . . . . . . . . . . . . . . . . 387

Masken zwischen Spiel und Ernst. Beiträge des Tübinger Arbeitskreises für Fasnachtsforschung. $\mathrm{Hg}$. von Hermann Bausinger (Siegfried Kube) . . . . . . . . . 388

Wolfgang Suppan, Volkslied. Seine Sammlung und Erforschung (Winfried Schrammek) . . . . . . . . . . . . . . . . . . . . . . . . . . 389

Sowjetische Volkslied- und Volksmusikforschung. Ausgewählte Studien. Hg. v. Erich Stockmann u. Hermann Strobach (Isolde Gardő) . . . . . . . . . 39i

Adolf Dygacz, Rzeka Odra w polskiej pieśni ludowej (Die Oder im polnischen Volkslied) (Wilhelm Szewczyk) . . . . . . . . . . . . . . . . . . . 394

GotTFRIEd Weissert, Das Mildheimische Liederbuch. Studien zur volkspädagogischen Literatur der Aufklärung (Waltraud Woeller) . . . . . . . . . . . 395

Maud Karpeles, Cecil Sharp. His Life and Work (Winfried Hoffmann) . . . . . . 396 
JøRn Pıø, Folkeminder og Traditionsforskning (Gisela Burde-Schneidewind) . . . . 398

Lutz RöHRich, Sage (Dies.) . . . . . . . . . . . . . . . . . . . . . . . . . 399

ERhard Hexelschneider, Die russische Volksdichtung in Deutschland bis zur Mitte des 19. Jahrhunderts (Herbert Peukert). . . . . . . . . . . . . . . . . . . . 400

Roman Jakobson, Selected Writings IV. Slavic Epic Studies (Ders.). . . . . . . . 402

Ovidiu BîrleA, Antologie de proză populară epică (Anthologie epischer Volksprosa) (Hanni Markel)

Ders.: Poveștile lui Creangă (Die Erzählungen Creangăs) (Dies.) . . . . . . . . . 405

Richard M. Dorson, American Negro Folktales (Friedmar Geißler). . . . . . . . 407

Schottische Sagen. Hg. v. Christiane Agricola (Gisela Burde-Schneidewind) . . . 408

Belorussische Volksmärchen. Hg. von L. G. Barag (Helena Kapełuś) . . . . . . . 409

ELEK BENEDEK, A táltos asszony. Népmesék és mondák (Die zauberkundige Frau.

Volksmärchen und Sagen) (Ágnes Kovács) . . . . . . . . . . . . . . 4II

Lisa Tetzner, Aus der Welt des Märchens (Siegfried Neumann) . . . . . . . . . 413

Arpád Antal, József Farag6, T. Attila Szabo, Kriza János. Három tanulmány

(Drei Studien) (Ágnes Kovács) . . . . . . . . . . . . . . . . . . 4 414

Südhessisches Wörterbuch. Begründet v. Friedrich MAUReR, bearb. v. RUDOLF Mulch (Anneliese Bretschneider) . . . . . . . . . . . . . . . 415

Wolfgang SpERbER, Die sorbischen Flurnamen des Kreises Kamenz (Ostteil) (Wilfried Fiedler) . . . . . . . . . . . . . . . . . . . . . 416

Dietmar Wünschmann, Die Tageszeiten. Ihre Bezeichnung im Deutschen (Heinz Gebhardt) . . . . . . . . . . . . . . . . . . . 418

Wege und Forschungen der Agrargeschichte. Festschrift zum 65. Geburtstag von Günther Franz. Hg. von Heinz Haushofer und Willi A. Boelcke (Ulrich Bentzien) . . . . . . . . . . . . . . . . . . . . . 419

R. C. Hekker und J. M. G. van der Poel, De nederlandske boerderij in het begin der rge eeuw (Hans-Heinrich Müller) . . . . . . . . . . . . . . . 420

UlRICH Thомas, Die landwirtschaftlichen Prämiierungen in Württemberg im I9. Jahrhundert (Ders.) . . . . . . . . . . . . . . . . . . . . . . . . . 420

HEINZ WIESE und JohanN Bölts, Rinderhandel und Rinderhaltung im nordwesteuropäischen Küstengebiet vom I g. bis zum 19. Jahrhundert (Ders.) . . . . . . 422

J. M. G. van der Poel, Oude Nederlandse ploegen (Ulrich Bentzien) . . . . . . . 423

JoSEF VAŘEKa, Vétrné mlýny na Moravé a ve Slezsku (Windmühlen in Mähren und Schlesien) (Karl Baumgarten) . . . . . . . . . . . . . . . . . 423

Alfred Fiedler - Jochen Helbig, Das Bauernhaus in Sachsen (Ders.) . . . . . 424

Rudolf Helm, Das Bürgerhaus in Nordhessen (Ders.) . . . . . . . . . . . . . 425

Hermann Kolesch, Das altoberschwäbische Bauernhaus (Ders.) . . . . . . . . . 427

Chronologie des künstlerischen Volksschaffens 1963-1966 (Ute Mohrmann) . . . . 428

LEOPOLD SCHMIDT, Volkskunst in Österreich (Manfred Bachmann) . . . . . . . . 429

LEOPOLD SCHMidT, Bauernmöbel aus Süddeutschland, Osterreich und der Schweiz

(Ute Mohrmann) . . . . . . . . . . . . . . . . . . . . . 430

Alexander K. Tschekalow, Bäuerliche russische Holzskulptur (Dies.) . . . . . . 43 I 
